\title{
DIGITALCOMMONS
}

@WAYNESTATE —

Wayne State University

Physics and Astronomy Faculty Research

Publications

Physics and Astronomy

9-21-1988

\section{A novel algorithm for calculating the time evolution of the electron energy distribution function in gaseous discharges}

P J. Drallos

Wayne State University

J M. Wadehra

Wayne State University, ad5541@wayne.edu

\section{Recommended Citation}

Drallos PJ, Wadehra JM. A novel algorithm for calculating the time evolution of the electron energy distribution function in gaseous discharges. J. Appl. Phys. 1988;63(11):5601-5603. doi: 10.1063/1.340340

Available at: http://digitalcommons.wayne.edu/phy_astro_frp/99 


\title{
A novel algorithm for calculating the time evolution of the electron energy distribution function in gaseous discharges
}

\author{
P. J. Drallos and J. M. Wadehra \\ Department of Physics and Astronomy, Wayne State University, Detroit, Michigan 48202
}

\begin{abstract}
(Received 12 November 1987; accepted for publication 22 January 1988)
We are presenting a novel numerical technique for obtaining the time evolution of the electron velocity and electron energy distribution functions in the presence of a uniform electric field. Using this technique, the various swarm parameters can be evolved for sufficiently long times so that equilibrium can be reached without incurring any rumerical instabilities. Results are presented for electron swarms in gaseous neon for various values of $E / N$.
\end{abstract}

In nearly all aspects of gaseous electronics, the electron energy distribution is of fundamental importance. Knowledge of the electron energy distribution function (EEDF) is usually gained through its relation with the electron velocity distribution function (EVDF) which is a solution of the Boltzmann equation for a given set of collision cross sections. The Boltzmann equation can be solved analyticaliy for an EEDF for only a few simple cases. 'In practice, however, numerical methods have to be used for its solution. In traditional techniques for solving the Boltzmann equation, the distribution function is expanded in the Legendre polynomials-either a two-term expansion or a multiterm expansion-and the resulting set of equations are solved numerically for the equilibrium EEDF after making some simplifying approximations for the collision cross sections.

However, these expansion techniques do have some in. herent drawbacks. ${ }^{2}$ For example, the two-term expansion method breaks down for high values of $E / N$ but works well for low $E / N$ values. The EEDF under high $E / N$ situations can, in principle, be obtained by taking more terms in the Legendre expansion of the Boltzmann equation. It is not always clear when the multiterm expansion is to be preferred over the two term, nor just how many terms in the expansion of the EEDF are to be included. The convergence behavior of a multiterm expansion, especially for high values of $E / N$, is also not completely understood. Furthermore, the cross sections, which are adjusted to reproduce the experimental swarm parameters in a two-term expansion, do not yield the same EEDF or the corresponding swarm parameters when used with a multiterm expansion and vice versa. It would be desirable, then, to have a procedure for obtaining the equilibrium EEDF and equilibriun EVDF that does not involve legendre expansion of the distribution function. Here we have utilized a finite-difference scheme, previously used by Tagashira and co-workers, ${ }^{3,4}$ in which no expansion of the EVDF in spherical harmonics is needed and the time evolution of the EVDF to its equilibrium value is obtained explicitly. Thus, the question of how many terms should be taken in the expansion of the EVDF, which is the solution of the Boltzmann equation, is completely avoided.

In this communication we present a novel algorithm for obtaining the time evolution of the EVDF numerically. This is accomplished by beginning with the Boltzmann equation which can be written as

$$
\frac{\partial f(\mathrm{v}, t)}{\partial t}+\mathbf{a}^{\cdot} \nabla_{v} f(\mathbf{v}, t)=R(\mathbf{v}, t)
$$

where $a=-(e \mathrm{E} / \mathrm{m})$ is the acceleration of the electrons of mass $m$ due to the electric field $R$, and $R(\mathrm{y}, t)$ is the collision term containing all of the relevant cross sections. Now we multiply both sides of $\mathrm{Eq}$. (1) by a finite time interval $\Delta t$ and then add $f(v, t)$ to each side which yields,

$f(v, t)+\left(\Delta t \frac{\partial}{\partial t}+\Delta v \cdot \nabla_{v}\right) f(\nabla, t)=f(v, t)+R(v, t) \Delta t$

with $\Delta v=a \Delta t$. The terms on the left-hand side of $\mathrm{Eq}$. (2) can be combined to yield the final result,

$$
f(v+\Delta v, t+\Delta t)=f(\psi, t)+R(v, t) \Delta t \text {. }
$$

Equation (3) describes the time evolution of the EVDF and can thus be used to obtain the time evolution of the EEDF and the various electron swarm parameters. Note that Eq. (3) is entirely equivalent to Eq. (1) and is perhaps even more fundamental than Eq. (1), as Eq. (3) is a necessary step that one must go through in a textbook derivation of the Boltzmann equation. ${ }^{5}$ In such a derivation, one would normally expand the ieft-hand side of Eq. (3) to first order in $\Delta t$, then take the limit $\Delta t \rightarrow 0$ to obtain the Boltzmann equation [Eq.(1)]. In the velocity space, the collision term $R(v, t)$ in $\mathrm{Eq} .(3)$ is difficult to evaluate in Cartesian coordinates, but straightforward in spherical coordinates since the collision cross sections depend only on the electron impact speed $v$. Thus, it might seem that spherical coordinates would be the natural choice for evaluation of Eq. (3). In their calculations, Kitamori, Tagashira, and Sakai ${ }^{4}$ used spherical coordinates to evaluate the term ${ }^{*}{ }_{w} f(\mathrm{v}, t)$ of Eq. (1). This involves derivatives of $f$ with respect to $v$ and $\theta$ (polar coordinates in velocity space) which must be evaluated numerically. This procedure leads to strong numerical instabilities in the EVDF and is computationally expensive.

On the other hand, as we will now show, $f(v+\Delta v, t+\Delta t)$ of Eq. (3) is extremely easy to evaluate in Cartesian coordinates. The acceleration of electrons along $\mathrm{E}$ (which we choose to be along the $z$ direction) is constant, and is zero in the transverse directions. Thus, in Cartesian coordinates, $\mathrm{Eq} .(3)$ is written as

$$
\begin{gathered}
f\left[v_{x}, v_{y}, v_{z}-(e E / m) \Delta t, t+\Delta t\right] \\
=f\left(v_{x}, v_{y}, v_{z}, i\right)+R(v, t) \Delta t
\end{gathered}
$$

Equation (4) is very well suited for evaluation on a computer. Since there is axial symmetry along $v_{z}$, fneed only be stored as a function of $v_{z}$ and $v_{x}$ (or $v_{y}$ ) in such a way that the velociry increments $\Delta v$ satisfy $\Delta v=(e E / m) \Delta t$. Evaluation 
$E / N=144 \mathrm{Td}$

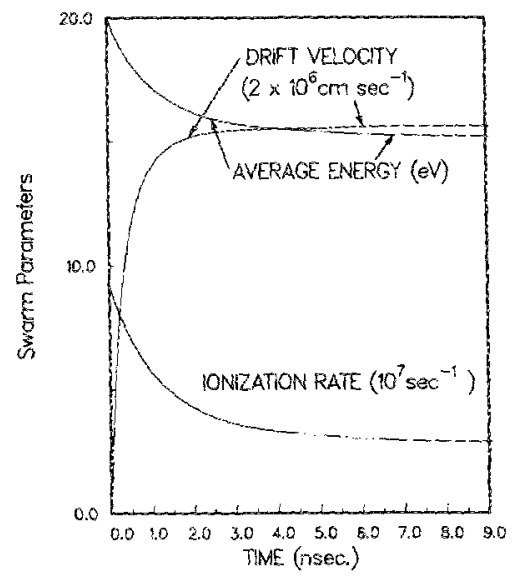

FIG. 1. The time evolution of various electron swam parameters in gaseous neon for $E / N=144 \mathrm{Td}$.

of $f$ in Eq. (4) then merely involves, in part, a shifting of the array $f\left(v_{x}, v_{z}\right)$ along $v_{z}$ at each time interval $\Delta t$. This shifting procedure accomplishes all the acceleration effects of the electrons due to the electric field without incurring the related problems of numerical instabilities which arise mainly from the evaluation of derivatives. The evaluation of the col. lision term ${ }^{4}$ in $\mathrm{Eq}$. (4) involves an integral over the polar angie $\theta$ (in the velocity space) and requires a knowledge of the distribution function at various values of $v$ and $\theta$, that is, at various $v-\theta$ grid points in the velocity space. This integra. tion can be carried out, even though $f$ is known only as a function of $v_{x}$ and $v_{z}$, by simply interpolating $f\left(v_{x}, v_{z}\right)$ to get $f(v, \theta)$.

Thus, the novel algorithm for evaluating the time evolution of the EVDF is as follows: Starting from a distribution function at some time $t$ (a Maxwellian at $t=0$, for example) which is stored in a wo-dimensional array $f\left(v_{x}, v_{z}\right)$ such that $\Delta v=(e E / m) \Delta t$, the collision terms $R\left(v_{x}, v_{z}\right)$ for each $v_{x}$ and $v_{z}$ are calculated. These collision terms are then multiplied by $\Delta t$ and added to the distribution function from which they were obtained in accordance with Eq. (4).

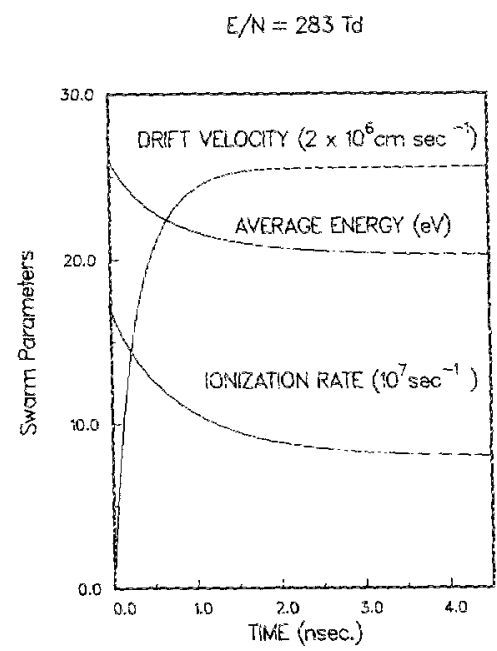

FIG. 2. The time evolution for various electron swarm parameters in gaseous neon for $E / N=283 \mathrm{Td}$.

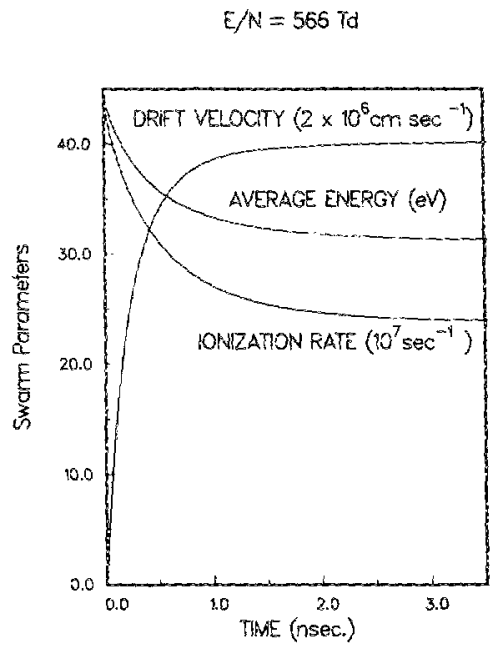

FIG. 3. The time evolution for various electron swarm parameters in gase ous neon for $E / N=566 \mathrm{Td}$.

The resulting array is then shifted along the $v_{z}$ index $\left[f\left(v_{x}, v_{z}\right) \rightarrow f\left(v_{x}, v_{z}+\Delta v\right)\right]$ and it becomes the new distribution function at the later time $t+\Delta t$. This procedure is repeated while various swam parameters are calculated from each new distribution function corresponding to a new time $t+\Delta t$. Equilibrium is obtained when the swarm parameters cease to change in time.

We have used this method to obtain the time evolution of various electron swarm parameters for the electron-neon system for various values of $E / N$. In all of our calculations we assumed an initial Maxwellian velocity distribution at $t=0$ and a gas density $N$ of $3.54 \times 10^{+16} \mathrm{~cm}^{-3}$ or $1.32 \times 10^{-3}$ amagat $(1$ Torr at $273 \mathrm{~K}$ ). The velocity steps $\Delta v$ ranged from $2.2 \times 10^{7}$ to $3.4 \times 10^{7} \mathrm{~cm} / \mathrm{s}$ and the time steps $\Delta t$ ranged from 0.064 to 0.032 ns as $E / N$ was varied from 144 to $566 \mathrm{Td}$. We point out that, in practice, $\Delta t$ may be chosen to

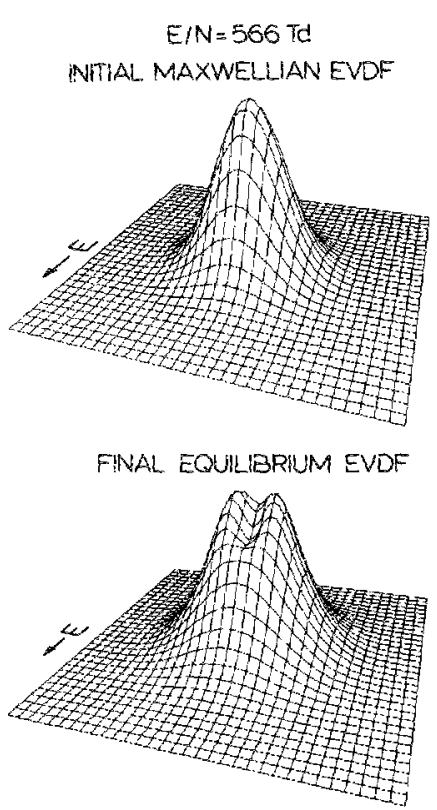

FIG. 4. The initial Maxwellian and final equilibrium electron velocity distribution function in neon for $E / N=566 \mathrm{Td}$. 
be a proper fraction of $m \Delta v / e E$ and only this fraction of each element of the EVDF is shifted per time step. Such a procedure is observed to enhance the numerical stability. Another criterion for choosing $\Delta t$ is that the term $R(v, t) \Delta t$ of $E q .(4)$ be smaller than $f(v, r)$ for all values of $v$. Figures $1-3$ display the calculated time-dependent behavior of various electron swarm parameters in gaseous neon for three different values of $E / N$. The relevant collision cross sections were taken from Ref. 4. The equilibrium values of these swarm parameters are in very good agreement with those calculated by Kitamori, Tagashira, and Sakai. ${ }^{4}$ We have also noted that the final equilibrium values of the swarm parameters are unaffected by the average energy value of the initial velocity distribution, although the transient behavior may be somewhat different. For example, an overshoot in the drift velocity is observed if the initial average energy of the discribution function is less than the final equilibrium value of the energy.
Figure 4 shows the initial and fnal EVDF for the case $E / N=566 \mathrm{Td}$.

The FORTRAN 77 code which we used was less than 400 lines in length and required about $500 \mathrm{CPU}$ seconds to reach equilibrium for $E / N=566 \mathrm{Td}$ on an $\mathrm{Amdahl} 470 / \mathrm{V} 6 \mathrm{man}$ frame computer.

The support of the U.S. Air Force Offce of Scientific Research through Grant No. AFOSR-87-0342 is gratefully acknowledged.

${ }^{1}$ L. C. Pitchford, Technical Report No. AFWAL-TR-85-2016 (1985).

${ }^{2}$ L. C. Pitchford, S. V. O'Neil, and J. R. Rumbie, Jr., Phys, Rev. A 23, 294 (1981).

${ }^{3}$ H. Tagashira, Y. Sakai, and S. Sakamoto, J. Phys. D 10, 1051 (1977).

${ }^{4} \mathrm{~K}$. Kitamori, H. Tagashira, and Y. Sakai, J. Phys. D 11, 283 (1978).

'K. Kuang, Statistical Mechanics (Wikey, New York, 1963), p. 57.

\title{
Distributed Bragg reflector $\mathrm{Pb}_{1-x} \mathrm{Sn}_{x} \mathrm{Se} / \mathrm{Pb}_{1-x-y} \mathrm{Eu}_{y} \mathrm{Sn}_{x}$ Se diode lasers with a broad single-mode tuning range
}

\author{
Y. Shani, R. Rosman, and A. Katzir \\ School of Physics and Astronomy, Tel Aviv University, Tel Aviv 69978, Israel \\ P. Norton, M. Tacke, and H. M. Preieral \\ Fraunhofer-Institut fuer Physikalische Messtechnik, Heidenhofstrasse $8,0-7800$ Fretburg. \\ Federal Republic of Germany
}

(Received 3 September 1987; accepted for publication 25 January 1988)

Distributed Bragg reflector $\mathrm{Pb}_{1,-x} \mathrm{Sn}_{x} \mathrm{Se} / \mathrm{Pb}_{1-x-y} \mathrm{Eu}_{y} \mathrm{Sn}_{x} \mathrm{Se}$ double heterostructure stripe geometry diode lasers were fabricated using molecular-beam epitaxy. Single-mode $\mathrm{cw}$ operation at about $7.8 \mu \mathrm{m}$ was obtained for heat-sink temperatures in the range $25-75 \mathrm{~K}$. the single-mode continuous tuning range was $10 \mathrm{~cm}^{-1}$. Tuning the diodes via the injection current, a range of $24 \mathrm{~cm}^{-1}$ was completely covered with single-mode emission. The reason for this wide turing range was mode hopping to lower frequencies rather than the usual hopping to higher frequencies.

Continuous tuning of single-mode $\mathrm{Pb}$-salic cw lasers over a broad range of frequencies is highly desired for ultrahigh resolution spectroscopy and for heterodyne systems. In the common Fabry-Perot (FP) lasers, continuous single-mode tuning can be obtained over a limited range of $1-2 \mathrm{~cm}^{-1}$. Broader ranges were achieved with cleaved coupled cavity $\left(C^{3}\right)$ lasers $\left(2.2 \mathrm{~cm}^{-1}\right.$ in Ref. 1$)$ and short cavity lasers $(6$ $\mathrm{cm}^{-1}$ in Ref. 2). As the current (or temperature) is increased in these lasers, the mode hopping to higher frequencies leaves gaps between the single-mode tuning ranges, and those gaps prevent a complete frequency coverage with single-mode operation.

The widest continuous turing range was obtained by distributed feedback (DFB) iasers, $7 \mathrm{~cm}^{-1} \mathrm{cw}$ in Ref. 3 , and $20 \mathrm{~cm}^{-1}$ in pulsed operation in Ref. 4. In a previous report,

\footnotetext{
3) Present address: Spectra Physies, Laser Analytics Division, 25 Wiggins Avenue, Bedford, MA.
}

single-mode cw operation of distributed Bragg reflector (DBR) lasers at about $77 \mathrm{~K}$ was described, with a continuous tuning range of $6 \mathrm{~cm}^{-1}$. We now describe DBR lasers with mode hopping to lower frequencies (rather than, as usual, to higher frequencies). A tuning range of $24 \mathrm{~cm}^{-1}$ is completely covered by single-mode $\mathrm{cw}$ operation in these lasers.

The laser chips were fabricated identical to those described in $R$ ef. 5 . Nevertheless, they differ in their size and in the way they were mounted on the cold post. Here we report on one such laser with an active layer $1.0 \mu \mathrm{m}$ thick having the $p-n$ junction removed $0.2 \mu \mathrm{m}$ from the active layer. The length of the active (pumped) region was $230 \mu \mathrm{m}$ and that of the passive (corrugated) region was $920 \mu \mathrm{m}$; the width of the lasers was $530 \mu \mathrm{m}$. This laser had a short active region and a long passive region, in order to suppress competing $F P$ modes. The passive section was mounted in good thermal contact with the cooled post [see Fig. 1(a)]. This was done 